\title{
A Comparative Analysis of Weed Images Classification Approaches in Vegetables Crops
}

\author{
Camilo A. Pulido Rojas ${ }^{\mathrm{a}, *}$, Leonardo E. Solaque Guzman ${ }^{\mathrm{b}}$, and Nelson F. Velasco Toledo ${ }^{\mathrm{c}}$ \\ Nueva Granada Military University, Bogotá, Colombia \\ E-mail: acamilopulidorojas@gmail.com (Corresponding author), bleonardo.solaque@unimilitar.edu.co, \\ cnelson.velasco@unimilitar.edu.co
}

\begin{abstract}
This paper exposes a comparative analysis of three weed classification strategies based on area and texture features over images of vegetable crops, focus on provide a technological tool to support farmers in their maintenance tasks. The classification alternatives embrace a basic approach which defines an umbral according to scene features, indeed, a detection with a certain degree of uncertainty on the decision region is purposed and a rigid boundary decision arrangement are exposed. A first mode carries out an unsupervised learning, it uses area and color features with a practical thresholding classifier to differentiate between weed and vegetable classes, the following two, extracts statistical measures of autocorrelation, contrast, correlation and others, from grey level cooccurrence matrices to calculate texture features, next, a principal component analysis is made for dimensionality reduction. These patterns serve as basis for training K-Nearest Neighbor and Support Vector Machine classifiers. The algorithms performance is measured calculating sensitivity (SN), specificity (SP), positive and negative predicted values (PPV and NPV), also, the execution time is stored and tabulated in order to evaluate the proposed methods. Finally, the results show a similar performance of correct classification over 90 and $80 \%$ on SN and SP indices respectively, however, approaches present a clear difference in execution time respect of train an evaluation stages.
\end{abstract}

Keywords: Weed classification, texture features, principal component analysis, support vector machines, k-nearest neighbor.

ENGINEERING JOURNAL Volume 21 Issue 2

Received 5 April 2016

Accepted 16 September 2016

Published 31 March 2016

Online at http://www.engj.org/

DOI:10.4186/ej.2017.21.2.81 


\section{Introduction}

Agricultural sector has pointing to environmental conditions to ensure quality and safety of crop production; this approach is based on technological development to support soil preparation, planting and weed removing processes. Particularly, among the most important crop problems lie in weeds growth, which increase the biological competition; causing a higher consumption of supplies such as fertilizers and water. Further, delays in product deliveries are generated, indeed, exists a considerable amount of working hours for inspection and weed removal. It is due to this task is tedious and limited to perform a crop sampling for weed and plagues diagnosis, therefore, 100 per cent of crop is not explored, consequently weeds and plagues are overlooked. Selective treatment and control of weed represent an opportunity for robotics applications, carrying out the inspection and removal of weeds without herbicides, resulting in a clean and appropriate way to maximize quality and soil performance, linked to the precision agriculture approach to support maintenance planning and decision making about crops. The process of selective weed removal performed by workers lies on move around vegetables crop making a visual inspection, bending and pulling weed plant, hence, an application that process crop images for weed detection can be solve problem exposed above. Machine vision modules has been a trend for researchers to perform weed detection getting coordinates to position an end-effector to make a mechanical removal task. Weed classification from outdoor images is complex due to random and uncontrolled light conditions, also, weed plants don't have a recognized patterns for distribution in crop, thus, size, shape and texture features has been used to purpose classifiers.

A considerable amount of literature has been published about weed detection, a work based on size and shape features to classify weed is exposed in [1]; they acquire crop images in RGB color space and uses the excessive green algorithm [2] to segment and separate plants, then, area, perimeter and longest chord features are calculated. Finally, with a threshold and crop masking made, weed is detected, however, authors do not discuss elapsed time to get an evaluation of the scene. Relating to colour and shape features, methods for segment plants aimed to perform on different sunlight and background conditions are Excess Green Index ExG [3] and Normalized Difference Vegetation Index [4]. Another research showed a real time system using Fast Fourier Transform and Grey level co-occurrence matrix for image processing with results between 70.4 and $72.5 \%$ classification accuracy [5], they do not expose computing time for each step of algorithm, but it can be inferred that works on 30fps with 640x480 image size. The authors in [6] investigated about Gabor Wavelets for texture descriptors [7] in order to distinguish broadleaf and grass, the classifier is based on a multilayer perceptron network and has an accuracy around $90 \%$, indeed, use images of $300 \times 250$ pixels and get a feature extraction time around 550 milliseconds and total scene evaluation near to $8 \mathrm{fps}$. In [8], researchers reviewed about weed classification based on texture features trying co-occurrence matrices (CCM) [9], to discriminate plants species with scales and orientation invariant leafs [10], also, they suggested to add additional features due to visible light spectrum, allowing extract 11 textural features [11], training a classifier for identify soil classes [12], achieving an accuracy of $93 \%$. An extension of CCM is the Level Co-Occurrence Matrix method [13], which it has been used for classification based on texture through calculation of features worked by Haralick [14], aimed at making training a neural network using ExG index [15] as pre-processing. Other classification algorithm bases his theory in $\mathrm{K}$ Nearest Neighbor (KNN), this method is worked in [16] where expose a comparison between 1 and 4 neighbors to leaf shape based plant species recognition getting around $92 \%$ of correct rate. Wu and Wen [17], proposed a Support Vector Machine SVM classifier to identify weeds in corn fields during early crop stages, using the co-occurrence matrix in gray levels and statistical histogram properties to extract texture features, making a dimensionality reduction using principal components analysis with greater accuracy to $92 \%$, nevertheless, training and evaluation times are not exposed.

Based on studies described above, exists a potential of using area-color and texture features to discriminate vegetables in crops using straightforward classification methods. The main contribution of the research presented in this paper is evaluate three strategies for weed classification from outdoor images with the same environmental conditions described as follows: The first approach is a basic methodology using direct features from plants images like area and color with a thresholding classifier. The following strategy for weed identification is based on soft clustering, this allows clusters overlap in feature space, measuring a degree of belonging to weed or vegetable classes, through statistical texture features extracted from Gray Level Co-occurrence matrices (GLCM) and kNN algorithm training. Finally, Support Vector Machine classifier is purposed as a hard clustering from the same texture patterns extracted in the previous strategy. 
This paper has been divided into five parts. The first part about Introduction, chapter 2 describes AreaColor features for weed classification. Texture features, dimensionality reduction and Support Vector Machine classifier, are explained in chapter 3. Next part is about results and conclusions. Finally, acknowledgements are exposed.

\section{Methodology}

The algorithms proposed use outdoor color images of vegetables crops. Samples of images for processing are shown in Fig. 1. Prior to commencing the study, theoretical foundation and process description is exposed as follows: first, feature extraction is exposed. In Subsection 2.1, low level features obtained directly from the image are explained, while, in Subsection 2.2, statistical texture features are showed with principal component analysis for dimensionality reduction. Next, decision equations and weed identification criteria are purposed for Thresholding (Section 2.31), KNN (Section 2.32) and SVM (Section 2.33) classifiers.
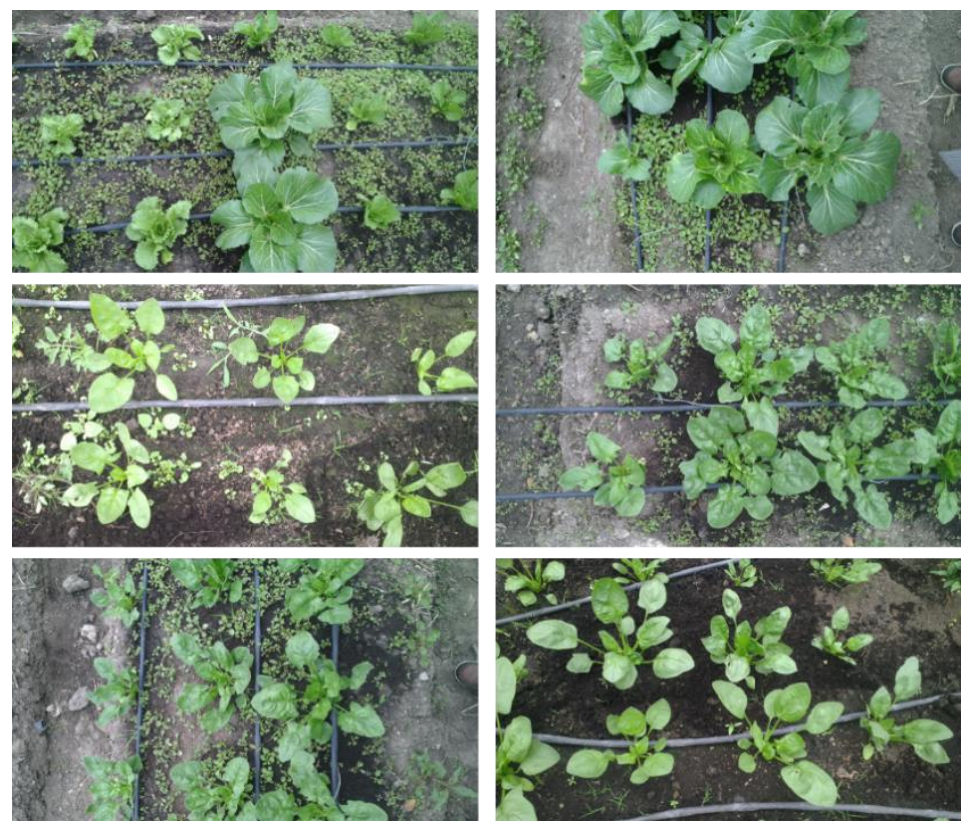

Fig. 1. Sample of crop images set.

\subsection{Low Level Features Extraction}

Due to random behavior of weed growing and relation with crop size during early ages, it is appropriate to use area and color features as descriptors for weed classification. These features are the most intuitive ways to detect weed in vegetable crops, therefore, the system purposed is a practical method to be used as binary classification separating soil and plants.

Algorithm begins by a Green Plant Detection result of evaluating the original grayscale image using Eq. (1). Next a Median filtering removes noise with advantage of preserving edges. Third, previous output is converted to binary, at this point, small objects are removed in order to avoid outliers.

$$
I_{\text {plant }}\left(X_{\text {pixel },}, Y_{\text {pixel }}\right)=I_{\text {Green Source }}\left(X_{\text {pixel }}, Y_{\text {pixel }}, G\right)-I_{\text {Gray }}\left(X_{\text {pixel }}, Y_{\text {pixel }}\right)
$$

Over the resulting image is appropriate to highlight objects in order to have a clear difference between soil and plants; this is accomplished by segmenting according to a threshold (Eq. (2)) defined by Otsu's method, with the purpose of estimates optimal value through an exhaustive search of maximum variance of gray levels. Additionally, results appropriate fill holes in the image, to enhance classification process with compact objects, to deal with this, an algorithm based on morphological reconstruction is used $[18,19]$. 


$$
I_{\text {Bin }}(X, Y)=\left\{\begin{array}{l}
0, I_{\text {Median }}(X, Y)<t \\
1, I_{\text {Median }}(X, Y) \geq t
\end{array}\right.
$$

The binary image contains groups of pixels that make up individual plants, therefore, it is necessary label objects in the scene to get a region for extract features. Labeling process is based on connected components algorithm with a 4 connectivity given a heuristic stated on pixel values according predecessor labels at north and west position exposed in $[20,21]$. The feature extraction step is carry out by defining labeled objects area, counting number of pixels for each one and storing values for all items [22]. The steps described above are shown as summary in Fig. 2.
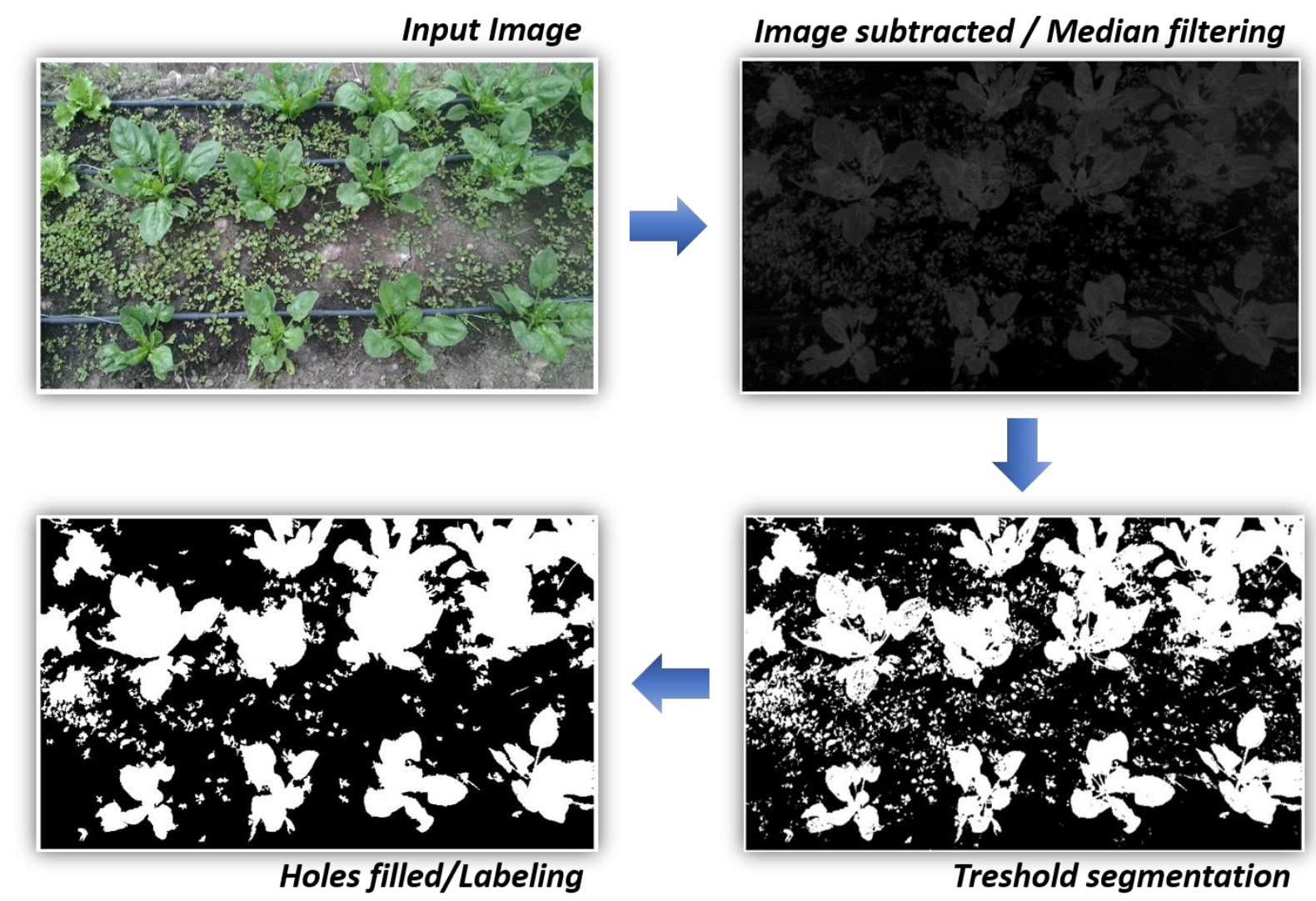

Fig. 2. Scene or crop image example.

\subsection{Statistical Features Extraction}

The issue of weed detection can also be focus on texture features; texture term refers to similarity and difference between high and low brightness values also called grey levels. For each evaluation scene, a Grey Level Co-Occurrence Matrix is calculated. Each resulting matrix serves as a basis to compute 10 statistical texture measures, therefore, a 10 dimensions feature space is constructed. After, principal component analysis (PCA) is used to represent original data in a new base where most variance is preserved on each axis; as a result, dimensionality reduction is performed to get a $2 \mathrm{D}$ feature space. Finally, patterns obtained are used to train classification algorithms based on K-nearest neighbor and Support Vector Machines. The descriptors must contain the most information possible about classes to discriminate them. In the same way, this corresponds to the direction with the greatest variance in the data. In practice, the classification accuracy increases as the dimensionality of the data is reduced. Figure 3 shows flow chart corresponding to process for validate the classification based on texture features. 


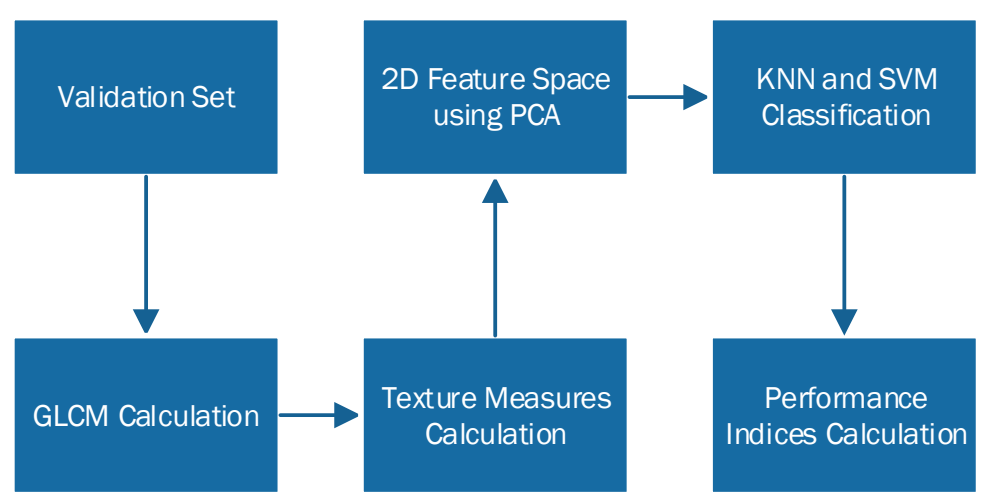

Fig. 3. Feature extraction flow chart.

\subsubsection{The gray-level co-occurrence matrix method}

The Gray-Level Co-Occurrence Matrix Method is a practical way to organize and tabulate the changes in brightness for different combinations of pixels, preserving the spatial information, getting first and second order texture measures, obtaining statistical calculations considering or not the relationship between neighboring groups. The mathematical definition of co-occurrence matrix is shown in the Eq. (3).

$$
C_{\Delta X, \Delta Y}(i, j)=\sum_{p=1}^{n} \sum_{q=1}^{m}\left\{\begin{array}{c}
1, \text { If } I(p, q)=i \text { and } I(p+\Delta x, q, \Delta y)=j \\
0, \text { Otherwise }
\end{array}\right.
$$

where $\mathrm{C}$ is a co-occurrence matrix defined over and I image with $\mathrm{m} \mathrm{x} \mathrm{n}$ size, parameterized with steps $\Delta \mathrm{x}$ and $\Delta \mathrm{y}$. The size of the matrix is based on divisions grayscale, for this paper, divisions correspond to 8 levels, obtaining an $8 \times 8$ matrix for each evaluation window. Due to texture calculations are weighted averages or statistics measurements of GLCM, it must be modified in such a way diagonal and normalized; therefore, the elements representing pairs of pixels on the diagonal have no difference in gray levels, in contrast, there are major differences in gray as the positions of matrix elements move away with respect to the diagonal. Present paper works with 10 texture measures computed from co-occurrence matrix, these features was selected for cover three groups: contrast, order and descriptive statistics. In addition, these measures allow to know similarity or local variance in the image, deviation of the gray levels, co-occurrence frequency of pixels, uniformity and homogeneity of the image within the image evaluation. The mathematical expressions about texture features are shown as follows:

\section{Autocorrelation:}

$$
\sum_{\mathrm{i}} \sum_{j}(i j) p(i, j)
$$

\section{Contrast:}

$$
\sum_{n=0}^{N g=1} n^{2}\left\{\sum_{i=1}^{N g} \sum_{j=1}^{N g} p(i, j)\right\}
$$

\section{Correlation:}

$$
\frac{\sum_{i} \sum_{j}(i, j) p(i, j)-\mu_{X} \mu_{X}}{\sigma_{X} \sigma_{Y}}
$$

Dissimilarity:

$$
\sum_{\mathrm{i}} \sum_{j}|i-j| \cdot p(i, j)
$$

\section{Entropy:}

$$
\sum_{i} \sum_{j} p(i, j) \log (p(i, j))
$$




\section{Energy:}

Homogeneity:

$$
\sum_{i} \sum_{j} p(i, j)^{2}
$$

$$
\sum_{\mathrm{i}} \sum_{j} \frac{1}{1+(i-j)^{2}} p(i, j)
$$

Variance:

\section{Difference Variance:}

$$
\sum_{i} \sum_{j}(i-\mu)^{2} p(i, j)
$$

\section{Cluster Shade:}

$$
\text { Variance } p_{X-Y}
$$

$$
\sum_{\mathrm{i}} \sum_{j}\left(i+j-\mu_{X}-\mu_{Y}\right)^{3} p(i, j)
$$

where $N_{X}$ and $N_{Y}$, are the columns and rows respectively to rectangular image, quantized in $N_{g}$ gray levels and $p(i, j)$ the input $(i, j) i t h$ normalized of GLCM.

\subsubsection{Principal component analysis and dimensionality reduction}

The principal component analysis (PCA) is a multivariate method or tool used to find patterns in data, establishing a relationship of observed variables to detect trends, groups, deviations and outliers, obtaining a representation of information, transformed by a linear combination that highlights their similarity and difference [23]. Therefore, it can be used as statistical characteristics extractor. The main advantage of using PCA is to find and compress patterns in data, causing a dimensionality reduction without much loss of information $[24,25]$. The objective of the analysis is to represent the data in terms of a $Y$ matrix, that contain the greatest variance information in the directions of their eigenvectors (Eq. (14)), from $X$ matrix with $n$ columns for samples and $m$ rows assigned to variables.

$$
\mathrm{Y}=\alpha \mathrm{X}
$$

The $\alpha$ vector are the directions of principal components. PCA considers to define independence of variance in the original data base, hence, this means de-correlate data finding directions, in which the variance is maximized and use it to define the new base [26]. Principal components result from eigenvectors of covariance matrix exposed in Eq. (15), and they are orthogonal to each other, so there is no redundant information. It should be noted, that the calculation of this matrix should be with the normalized data, subtracting the mean and divided by standard deviation (Eq. (16)), in order to avoid a large variance values due to measuring range and units of the extracted texture features.

$$
\begin{gathered}
\mathrm{C}_{X}=\frac{1}{n-1} X X^{T} \\
\mathrm{X}_{Z}=\frac{(X-\bar{X})}{\sigma}
\end{gathered}
$$

The eigenvectors are sorted in descending order respect to eigenvalues, which indicates the variance and importance of each principal component axis.

\subsection{Weed Classification}

Throughout this section, the classifiers purposed are discussed and linked with features described above. The Thresholding classifier uses area features for weed detection, while KNN and SVM classifiers focus on texture patterns result of principal component analysis to define decision rules; first one measures a degree 
of belonging to classes, whereas the other has a drastic border region to differentiate weed and vegetable classes.

\subsubsection{Thresholding classifier}

The objects labeled are sorted according to area values in descending order, when a difference with lower object is greater than $50 \%$, average of predecessors elements is calculated, this value is the threshold for weed detection. An example of weed identification result is shown in Fig. 4.

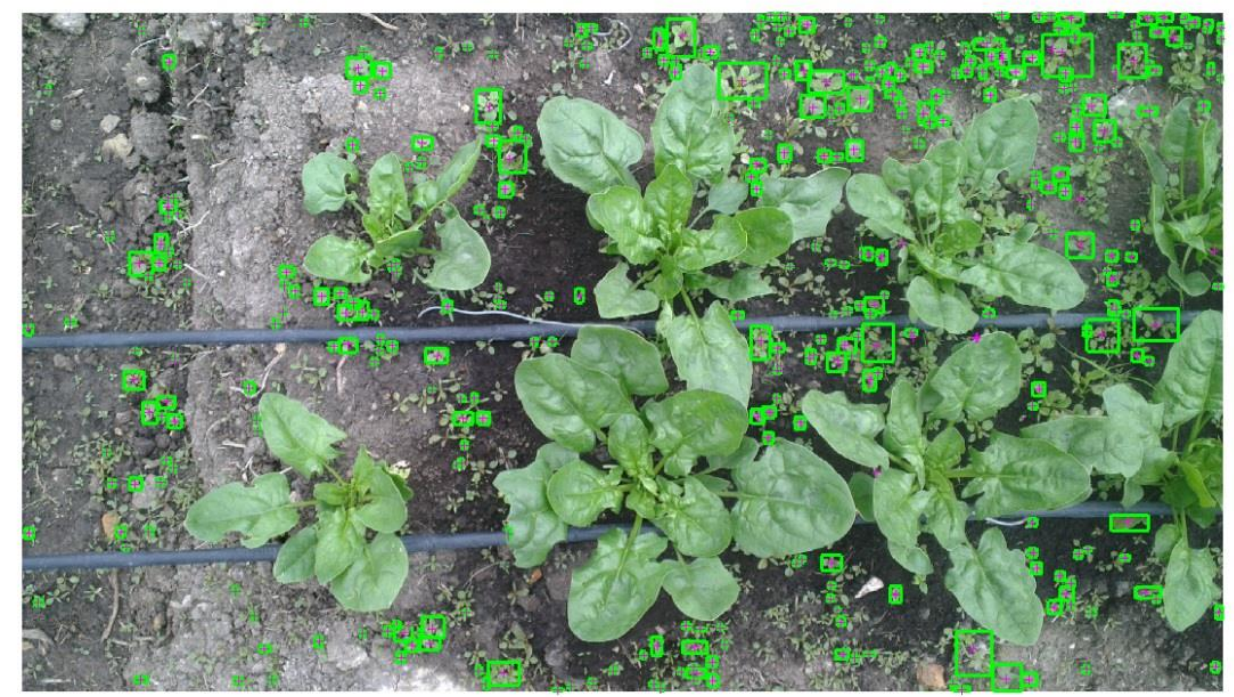

Fig. 4. Weed classification based on color and area features.

\subsubsection{K-nearest neighbor classification}

The k-nearest neighbor is a practical data mining algorithm and a standard nonparametric technique used for probability density function estimation and classification [27], then, using this classifier allows working texture features that do not obey a typical theoretical distribution assumptions. This method points out to search a group of $k$ objects in the training set that are closest to the validation data or new value, and bases the assignment of a label on the votation value according to classes in this neighborhood. Indeed, given a training set $\mathrm{T}$ and a new data for testing $u=\left(x^{\prime}, y^{\prime}\right)$, where $x^{\prime}$ are the coordinates in feature space and $y^{\prime}$ is its class assigned, the algorithm computes the distance between $u$ and each object in T, to get its nearestneighbor list $N N_{u}[28,29]$. The choice of the distance measure is relevant to improve accuracy. Criteria for selecting metric is focused on a small measure that produces a high likelihood of having the same class. Another point of interest is the $k$ number of neighbors to assess the class membership (See Fig. 5). If $k$ is large, the neighborhood includes points labeled in various classes, on the other hand, a small value output of algorithm can be sensitive to noise points. In practice, $k$ value can be adjusted using cross validation or if number of classes is equal to $2, k$ must be odd to avoid ties [30].

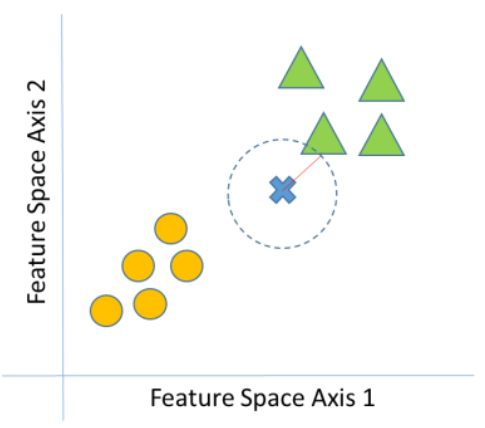

Fig. 5. Nearest Neighbor k=1. Triangles: Class $A$. Circles: Class B. X: Query Point. 
The classification is based on the majority class of its neighbors:

$$
\text { Majority Voting: } y^{\prime}=\operatorname{argmax}(L) \sum_{\left(x_{i}, y_{i}\right) \in N N_{u}} w_{i} x I\left(L=y_{i}\right)
$$

where $L$ is a class label, $y_{i}$ is the class label for the $i$ th nearest neighbors, and $I($.$) is a function that returns$ 1 if its argument is true and 0 otherwise. A more sophisticated approach, is taking reciprocal of the squared distance: $w_{i}=1 / d\left(x^{\prime}, x_{i}\right)^{2}$ aimed to give a weight to each object's vote by its distance [30].

\subsubsection{Support vector machine classification}

Support Vector Machine (SVM) is a discriminative classifier formally defined by a separating hyperplane. Support vectors are the examples closest to the separating hyperplane and the aim of SVM is to orientate this hyperplane in such a way as to be as far as possible (margin) from the closest members of both classes. This separating hyperplane works as the decision surface and is described by $w x+b=0$, where $\boldsymbol{w}$ is normal to the hyperplane and $b /\|w\|$ is the perpendicular distance from the hyperplane to the origin. The Figure 6 shows a decision boundary example for discriminate two classes A (Circles) and B (Triangles) corresponding to vectors $x_{i}$ of the training set, with $y_{i}$ class labels of +1 and -1 respectively. The hyperplane's equidistance from $\mathrm{H} 1$ and $\mathrm{H} 2(d 1+d 2)$ means a quantity known as margin.

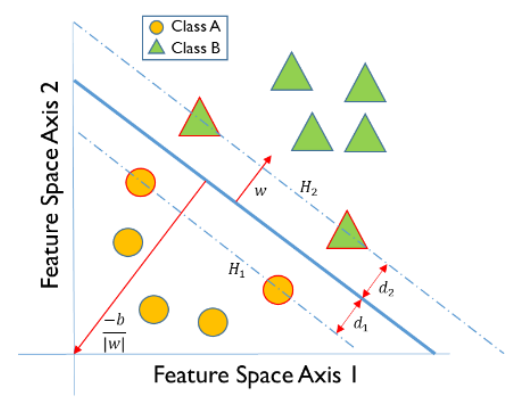

Fig. 6. Hyperplane through two linearly separable classes.

Then, the SVM approach is based on select the variables $\mathrm{w}$ and $\mathrm{b}$ to describe training data using Eq. (18)-(19).

$$
\begin{aligned}
& x_{i} \cdot w+b \geq+1 \\
& x_{i} \cdot w+b \geq-1
\end{aligned}
$$

In order to orientate the hyperplane to be as far from Support Vectors, is necessary to maximize the margin value: $1 /\|w\|$. This implies to:

$$
\begin{gathered}
\text { Minimize: } \emptyset(w, b)=\|w\|^{2} \\
\text { Subject to: } y_{i}[\langle w, x\rangle+b]-1 \geq 0
\end{gathered}
$$

This is a convex quadratic optimization problem, then can be expressed as a dual problem with Lagrange Multipliers.

$$
L(w, b, \Lambda)=\frac{1}{2}\|w\|^{2}-\sum_{i=1}^{l} \lambda_{i}\left[y_{i}\left(w \cdot x_{i}+b\right)-1\right]
$$

where $\Lambda=\left(\lambda_{1}, \ldots, \lambda_{l}\right)$ is the vector of non-negative Lagrange multipliers corresponding to the constraints in Eq. (22). Therefore, the dual problem is:

$$
\text { Maximize: } w(\lambda)=\sum_{i=1}^{l} \lambda_{i}-\frac{1}{2} \sum_{i=1}^{l} \sum_{j=1}^{l} \lambda_{i} \lambda_{i}
$$




$$
\text { Subject to: } \sum_{i=1}^{l} y_{i} \lambda_{i}=0, \lambda_{i}>0, i=1,2, \ldots l
$$

The optimal solution $\lambda^{*}$, is a discriminant function to classify new points in feature space. Equations 20 and 21 , shows how is built this function where $b^{*}$ is a threshold value [31].

$$
\begin{gathered}
b^{*}=y_{i}-w^{*} \cdot x_{i} \\
f(x)=\operatorname{sign}\left\{\sum_{i=1}^{l} y_{i} \lambda_{i}^{*}\left\langle x_{i}, x\right\rangle+b^{*}\right\}
\end{gathered}
$$

In most cases, the classification problems are nonlinear in feature space, then, SVM theory can be extended projecting input data to a higher dimensionality space, in which a separating hyperplane can be

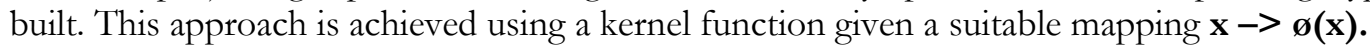

$$
f(x)=\operatorname{sign}\left\{\sum_{i=1}^{l} y_{i} \lambda_{i}^{*} \phi(x) \cdot \phi\left(x_{i}\right)+b^{*}\right\}
$$

\section{Results}

For the purpose of analysis, a crop image set was acquired, each outdoor image was captured by a person moving along vegetables crops using an $8 \mathrm{MP}$ camera. The photos are perpendicular to crop lines, and avoiding overexposure through manual adjustment of camera, therefore, the green components in images are preserved. The test crops are spinach and chard grown by Horticulture Technology, dependency of Nueva Granada Military University Campus in Cajica, Colombia. The crop image set is used for evaluate algorithms accuracy and time performance. The data processing system consist of a $3.30 \mathrm{GHz}$ processor and 8 GB RAM running MATLAB 2015b. It is an important component for classifiers purposed work with outdoor images, thus, additional computing time because of image pre-processing before classification stage is reduced. Indeed, data base was labeled manually based on random behavior of weed and the expertise of crops manager, in order to compare and evaluate the performance of the proposed area and texture approaches to weed detection.

The performance of classification algorithms was tested using sensitivity, specificity, positive predictive value and negative predictive value indices, calculated as follows:

\section{Sensitivity (SN):}

\section{Specificity (SP):}

$$
\frac{T P}{T P+F N}
$$

Positive Predicted Value (PPV):

$$
\frac{T N}{F P+T N}
$$

Negative Predicted Value (NPV):

$$
\frac{T P}{T P+F P}
$$

$$
\frac{T N}{F N+T N}
$$

where True positive $(T P)$ is the number of weeds detected as weed correctly. True negative $(T N)$ corresponding to the number of crops detected as crop correctly. False positive $(F P)$, the number of crop plants detected as weed and False negative $(F N)$, the number of weed plants detected as crop. A sample of cases described above for thresholding classifier is shown in Fig. 7, this is a part of a total image evaluation. The classifier draws a rectangle according to weed area and position, while the callouts indicate when occurs errors or successes in the detection stage. 


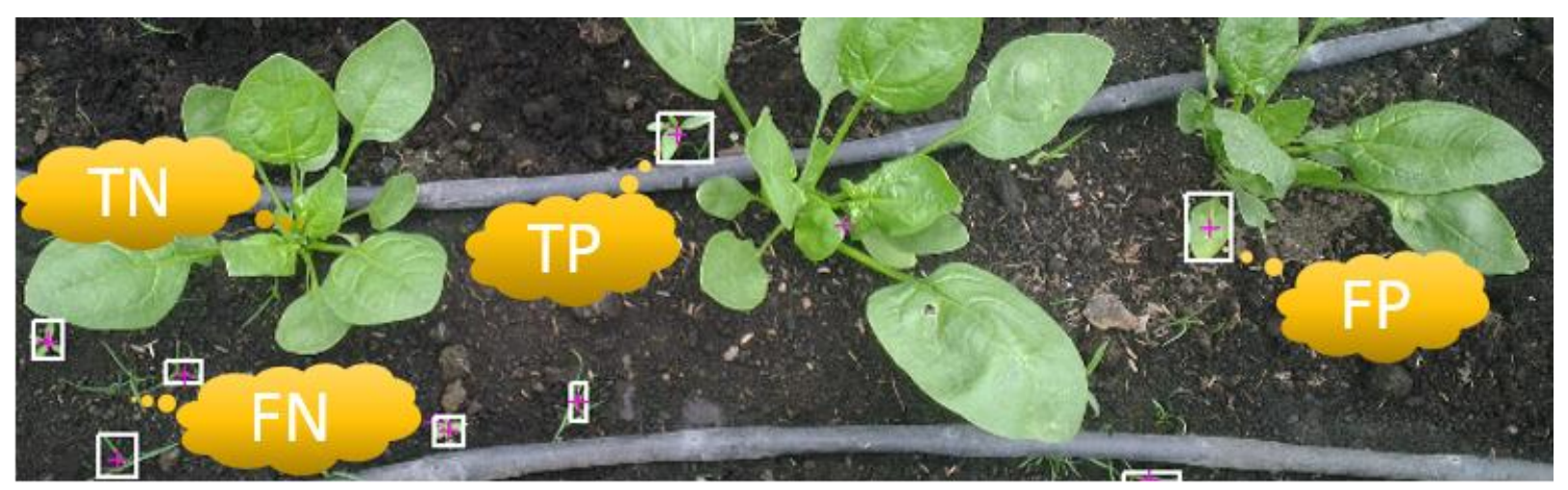

Fig. 7. Sample of TP, TN, FP and FP indices for thresholding classifier.

Meanwhile, the classifiers based on statistical texture features, result in a prediction value according to individual window evaluation, related with a class vector: 0 for weeds and 1 for plants, in this way, the training stages are carried out. The example shows in Fig. 8, represent a real case of TP, TN, FP and FN numbers as a result of $\mathrm{K}-\mathrm{NN}$ evaluation.

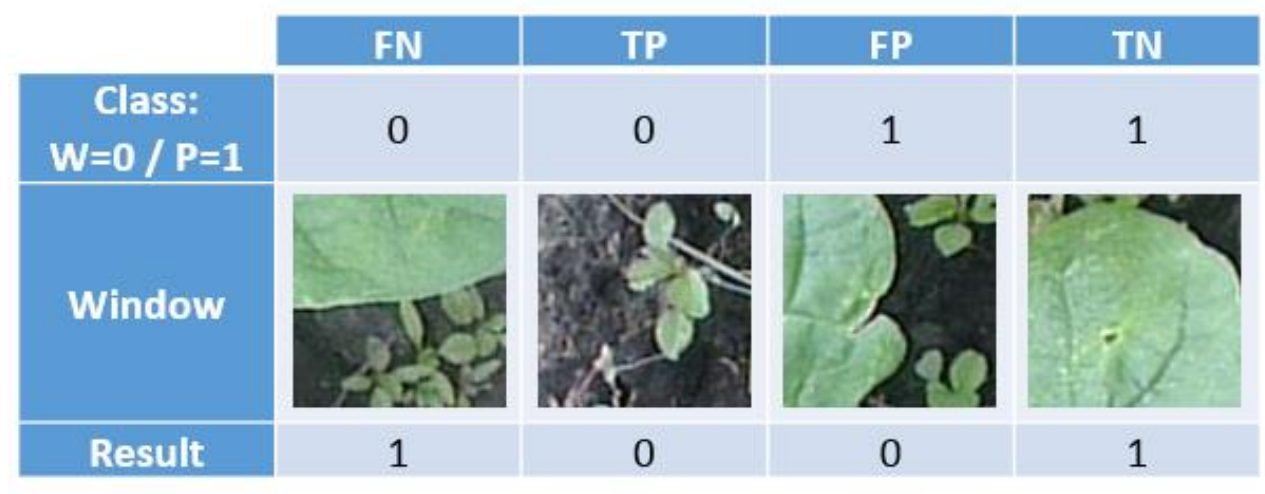

Fig. 8. Sample of TP, TN, FP and FP indices for texture-based classifiers.

\subsection{Classifiers Training}

Prior to commencing this subsection, results appropriate to highlight that thresholding classifier leads on unsupervised learning, consequently, only KNN and SVM training are exposed. From images set, each observation was labeled manually to identify weed and plants classes. The size of each observation is $100 \mathrm{x}$ 100 pixels. Some of the images used for feature extraction are shown in Fig. 9. 


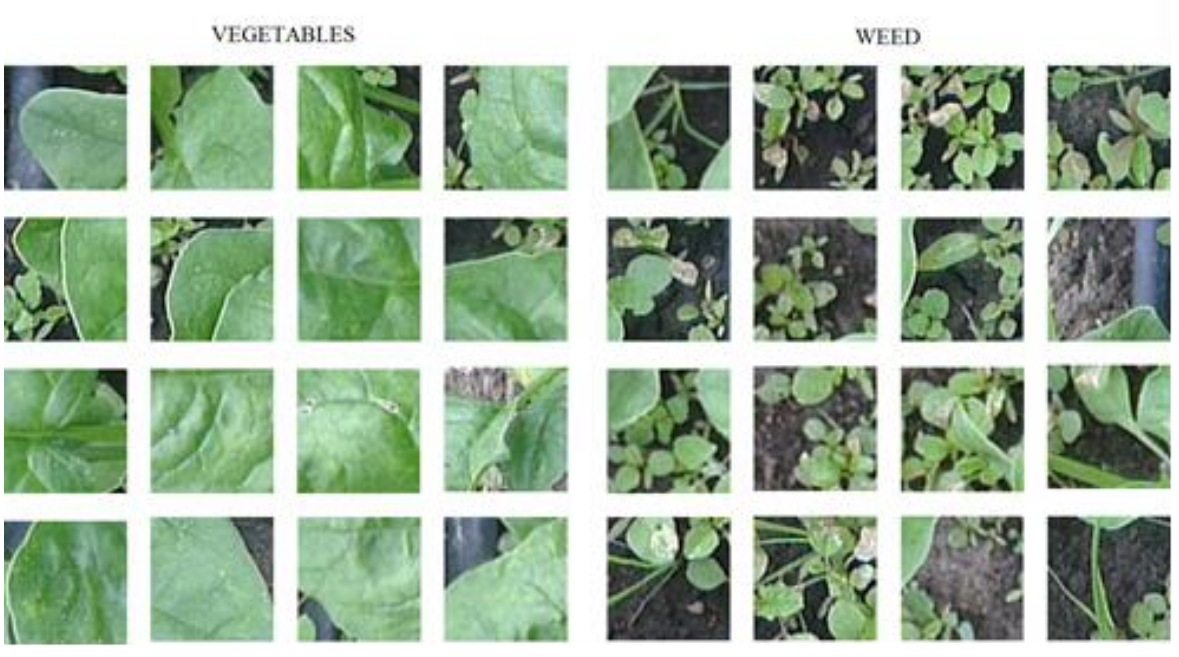

Fig. 9. Weed classification based on color and area features.

For the purpose of increase the reliability of weed classification, a dimensionality reduction is carried out preserving the greatest variance in the data, thus, texture descriptors are the principal components that best describe the features resulting from the linear combination of all calculated texture statistics.

The PCA calculation is performed on image set and the cumulative variances are shown in Table 1.

Table 1. Principal components cumulative variance (\%).

\begin{tabular}{cccccccccc}
\hline PC 1 & PC 2 & PC 3 & PC 4 & PC 5 & PC 6 & PC 7 & PC 8 & PC 9 & PC 10 \\
\hline 56.90 & 80.96 & 93.20 & 96.48 & 99.56 & 99.89 & 99.99 & 99.99 & 100 & 100 \\
\hline
\end{tabular}

The first two components retain $80.96 \%$ of the data variance. Figure 10 shows texture measurements mapped of these $2 \mathrm{D}$ components space.

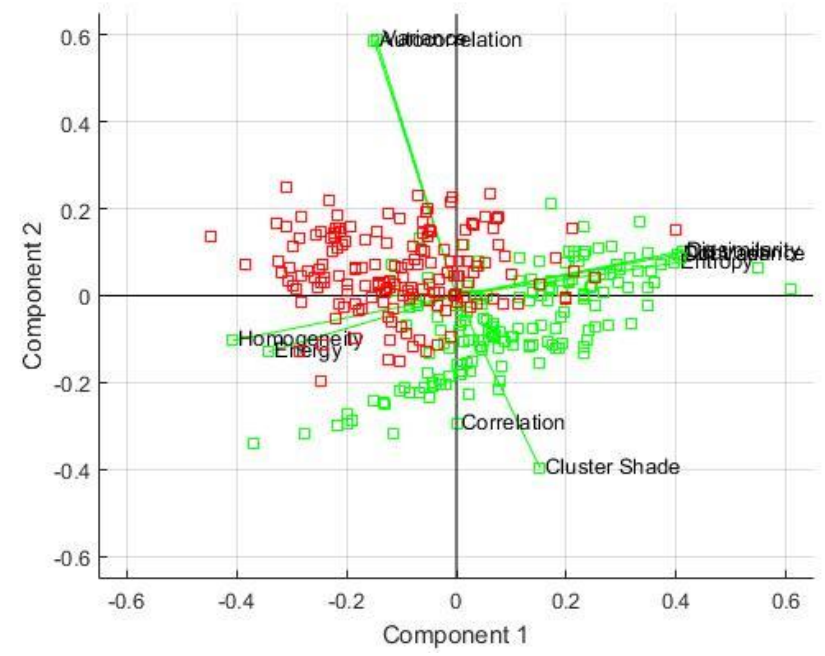

Fig. 10. Texture features plotted in 2D space—Red: weed; green: vegetables.

This result describes that data position in features space have a clear difference between weed and vegetables classes, thus, the classification step can be carried out because feature extraction was accomplished. 


\subsubsection{K-nearest neighbor training}

The training stage was carried out and validated using 10-Fold-Cross-Validation method, with 250 images, therefore, the test set contemplates 25 images on each algorithm evaluation. In this process, the results are presented as Boxplot in Fig. 11, according to Specificity SP, Sensitivity SN, Positive and Negative Predictive Value PPV - NPV indices calculated on each iteration.

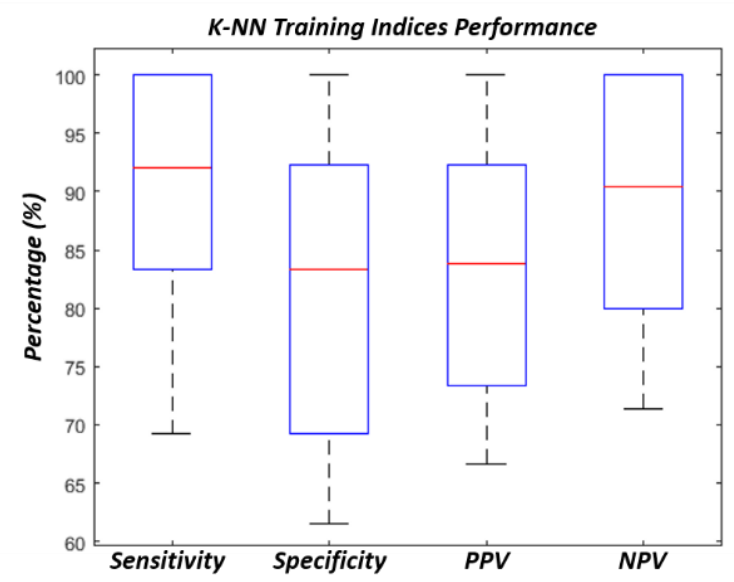

Fig. 11. K-NN Indices performance boxplot.

The classifier was validated using 70 images. Table 2 shows True Positive TP, True Negative TN, False Positive FP, False Negative FN and performance indices. The result reported more than $85 \%$ of correct classification.

Table 2. K-nearest neighbor validation result.

\begin{tabular}{cccccccc}
\hline TP & TN & FP & FN & SN & SP & PPV & NPV \\
\hline 33 & 30 & 5 & 2 & 94.286 & 85.714 & 86.842 & 93.75 \\
\hline
\end{tabular}

\subsubsection{Support vector machine training}

The highlighted support vectors (black circles) exposed in Fig. 12. Corresponding to training set results from dimensionality reduction using PCA with texture features.

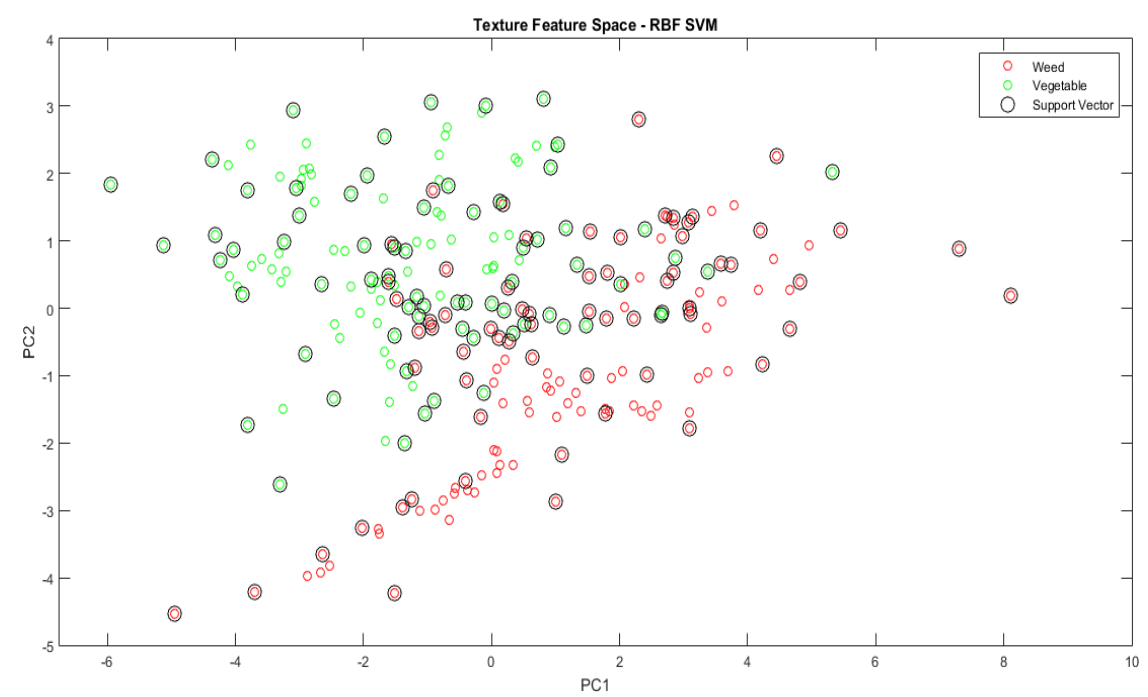

Fig. 12. Support vectors—Red: weed; green: vegetables; black circles: support vectors. 
The radial basis function (RBF) is used as kernel to resolve weed classification problem due to the nonlinear representation of texture features in principal components space. The mathematical definition of RBF is exposed in Eq. (28).

$$
k(x, y)=e^{-\|x-y\|^{2} / 2 \sigma^{2}}
$$

Figure 13 shows the contour of decision boundary corresponding to RBF kernel with $\sigma=1$ in training set.

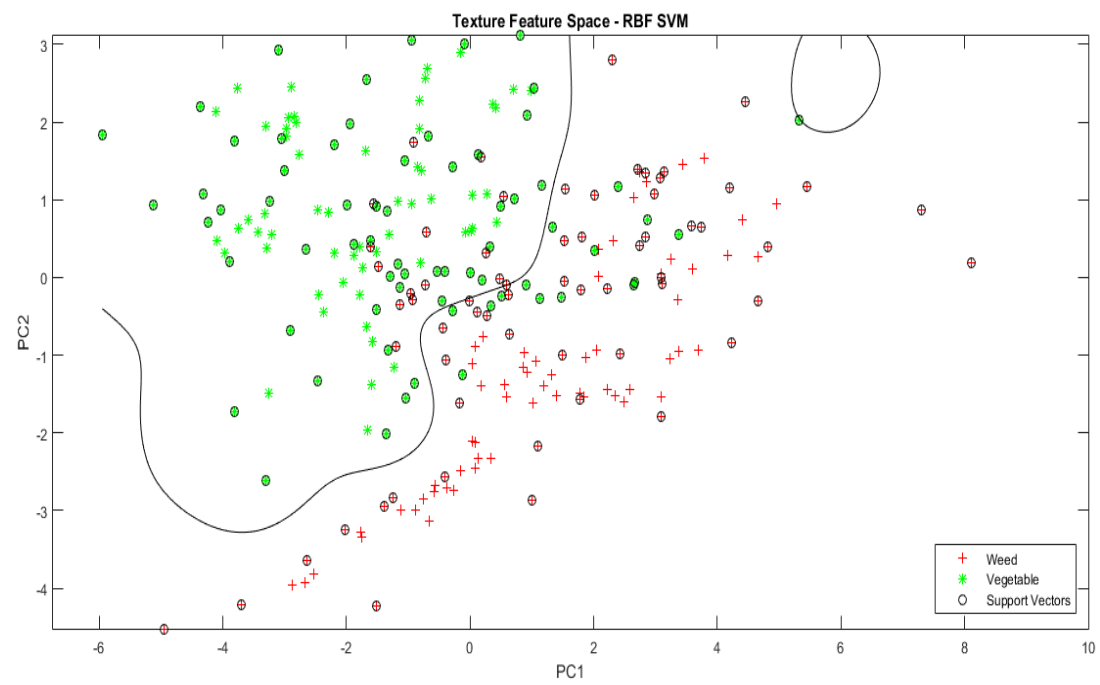

Fig. 13. Support vector machine classifier; initial decision boundary—Red: weed; green: vegetables; black line: decision boundary.

To increase the reliability of weed discrimination, the support vector machine trained above is optimized. For this purpose, the train data is partitioned into 10 set, then, 10 -fold cross-validation loss is expressed as function and it is used to find an optimal $\sigma$ value with the simplex search method [32]. The resulting value of $\sigma$ is 0.9614 and the smoothed decision boundary is shown in Fig. 14 .

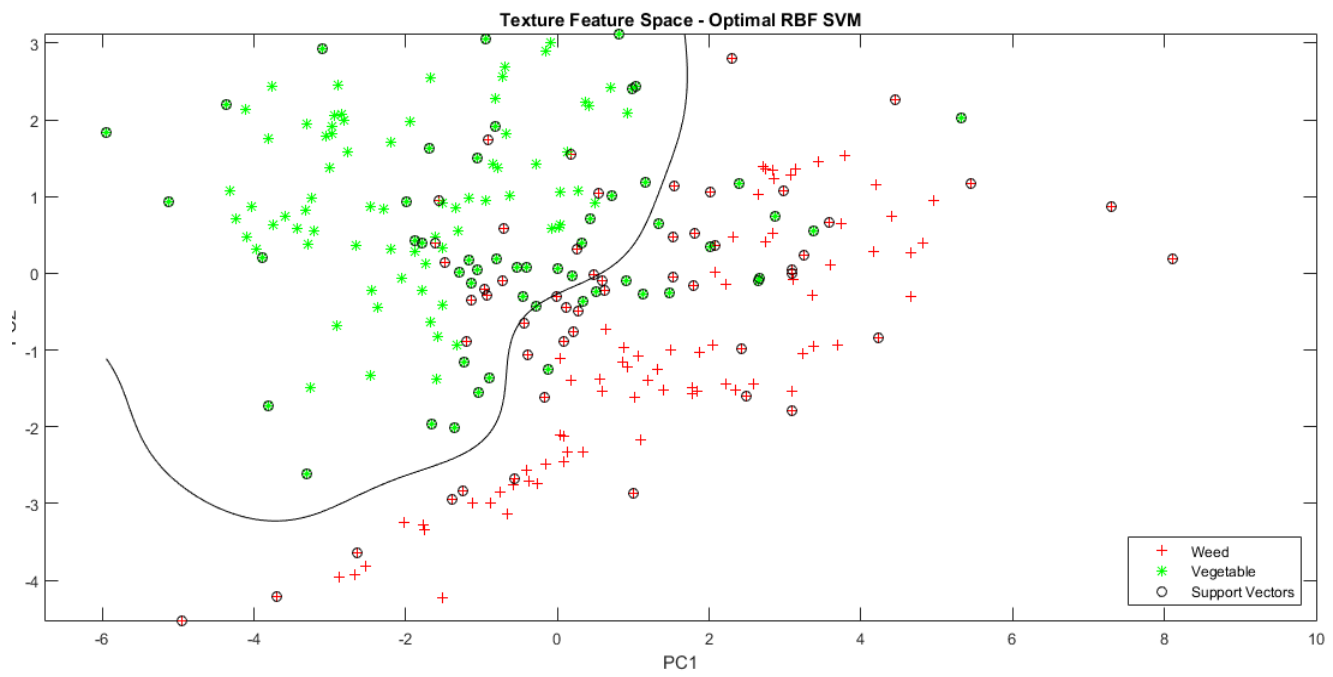

Fig. 14. Support vector machine classifier; smoothed decision boundary—Red: weed; green: vegetables; black line: decision boundary.

Such as K-Nearest Neighbor, the SVM classifier training was carried out with 250 training-test images and validated with 70 samples. The training uses 10-Cross-Fold-Validation method and the results are shown in Fig. 15, each box corresponds to the calculation of SN, SP, PPV and NPV indices on each 
iteration. It must be highlighted that each algorithm evaluation proceeds to optimize the decision boundary according to training data in this step.

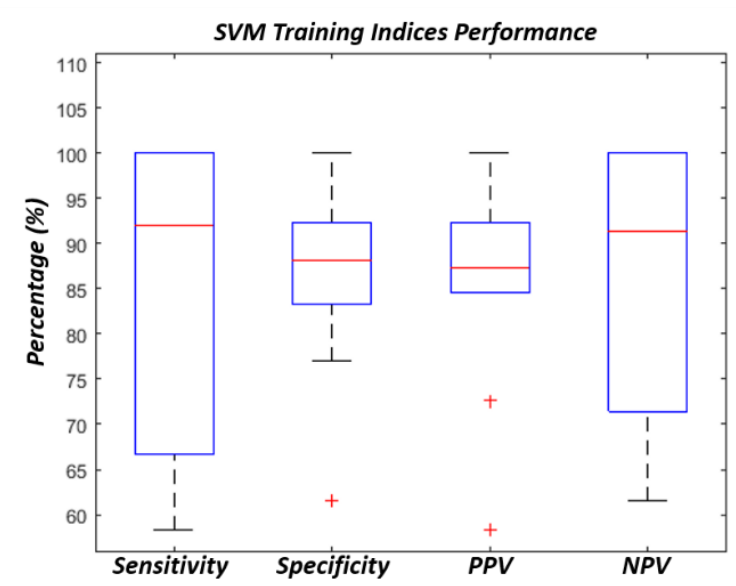

Fig. 15. K-NN indices performance boxplot.

The validation stage gets more than $94 \%$ of correct classification, the Table 3 counts TP, TN, FP and FP numbers, as well as performance indices.

Table 3. Support vector machine validation result.

\begin{tabular}{cccccccc}
\hline TP & TN & FP & FN & SN & SP & PPV & NPV \\
\hline 34 & 33 & 2 & 1 & 97.143 & 94.286 & 94.444 & 97.059 \\
\hline
\end{tabular}

\subsection{Strategies Comparison}

The performance of weed classifier based on area involves time measurements according to relevant steps of algorithm, this means, green detection, labeling and area thresholding, likewise, the indices described above are calculated and tabulated in order to get a system overall response using test images with 340 samples between weed and plants. The experiments were carried out over set with a size of 320 samples, labeled and stored respect their class in a column vector, with regard of classification performance indices calculations. The Table 4 below compares the experimental results tabulated as performance indices calculations.

Table 4. Performance indices calculation.

\begin{tabular}{|c|c|c|c|c|c|c|c|c|}
\hline $\begin{array}{l}\text { Binary } \\
\text { classifier }\end{array}$ & $\mathbf{T P}$ & $\mathbf{T N}$ & FP & FN & SN & SP & PPV & NPV \\
\hline $\begin{array}{l}\text { Area- } \\
\text { Based }\end{array}$ & 267 & 38 & 8 & 27 & 90.82 & 82.61 & 97.09 & 58.46 \\
\hline $\begin{array}{l}\text { KNN } \\
\text { Texture- } \\
\text { Based }\end{array}$ & 149 & 137 & 23 & 11 & 93.13 & 85.63 & 86.63 & 92.57 \\
\hline $\begin{array}{l}\text { SVM } \\
\text { Texture- } \\
\text { Based }\end{array}$ & 145 & 143 & 17 & 15 & 90.63 & 89.38 & 89.51 & 90.51 \\
\hline
\end{tabular}

This table is quite revealing in several ways, for instance, the response rate of correct classification was greater than $80 \%$ in all cases according specificity and sensitivity indices, being K-Nearest Neighbor classifier the best respect of sensitivity, which has a high ability to correctly identify plants as weed, whereas, the algorithm based on Support Vector Machines had the highest specificity value, around $90 \%$, indicating a great reliability for classifying plants within crop or vegetables class. On the other hand, area-based 
algorithm has the highest rate of PPV, showing that most of cases identified as weeds were true positives, although, it has the lowest percentage about NPV rate, resulting in the highest number of false negatives.

To assess proposed classifiers, the computing time is also measured in their respective stages (See Table 5). For supervised learning algorithms, the training and feature extraction time is tabulated. It is relevant to highlight that image size is $3264 \times 1840$ pixels and the Total column corresponds to time between read a source image and classification response, including feature extraction, arrange information in vectors and evaluation according to crop or weed classes.

Table 5. Measuring computing times of proposed algorithms in seconds.

\begin{tabular}{|c|c|c|c|c|}
\hline $\begin{array}{c}\text { Binary } \\
\text { classifier } \\
\text { Area- }\end{array}$ & $\begin{array}{c}\text { Green } \\
\text { Detection }\end{array}$ & $\begin{array}{l}\text { Labeling } \\
\text { and Area } \\
\text { Measure }\end{array}$ & Evaluation & Total \\
\hline Based & 0.0366 & 0.2677 & 0.065 & 0.64 \\
\hline \multirow{2}{*}{$\begin{array}{c}\text { KNN } \\
\text { Texture- } \\
\text { Based }\end{array}$} & \multirow[t]{2}{*}{$\begin{array}{c}\text { Texture } \\
\text { Features } \\
\text { PCA }\end{array}$} & Train & Evaluation & \multirow[t]{2}{*}{4.043} \\
\hline & & 0.19 & 0.0044 & \\
\hline $\begin{array}{c}\text { SVM } \\
\text { Texture- } \\
\text { Based }\end{array}$ & 4.125 & 124.170 & 0.0055 & 4.1632 \\
\hline
\end{tabular}

As can be seen from table, the algorithm with the lowest computing time is area-based, KNN and SVM are considerably slow to deliver the result of scene evaluation. Comparing the feature extraction times, the computational cost of texture statistical calculations is high, due to GLCM's and 2D feature space transformations, around 4.125 seconds to finish this task, whereas, area-based algorithm spends 0.3 seconds on pattern extraction, however, evaluating observations with supervised learning algorithms are up to 14 times faster than area thresholding. It is worth noting that there is a glaring difference between KNN and SVM training time, since, SVM includes the time necessary to optimize decision boundary or margin in a nonlinear way to adjust for best discrimination between classes in accordance with training sets.

\section{Conclusions and Future Work}

In general, classification algorithms should reach a balance between specificity and sensitivity values depending on application. Particularly, weed identification solutions focus on provide a basis for weed removal application, the system response must be reliable, avoiding take out vegetables plants.

This study was designed to compare three approaches for classify weed and vegetables. The methods purposed use outdoor images without preprocessing. A first mode carries out an unsupervised learning, it uses area and color features with a practical thresholding, the other two, perform texture features extraction and dimensionality reduction obtaining a patterns that best describes classes keeping most of the data variance from the co-occurrence matrix in gray levels to calculate texture features. These patterns serve as basis for training K-Nearest Neighbor and Support Vector Machine classifiers.

The results of this study suggest that the proposed algorithms for weed detection have a high performance and accuracy validated with sensitivity and specificity indices above 90 and $82 \%$ respectively. Therefore, a first approach to select the best with respect to these indices, such as the possibility of having a greater number of false negatives, because of robotic applications for weed detection are less critical if weeds are overlooked and not given as false positives where the system would removal vegetable plants. It should be noted and taking into account only the classification performance, independent of the computation time, algorithm based on SVM presented a best performance with specificity index around $90 \%$; it implies that number of vegetables identified as a weed is low, therefore, the reliability of the classifier would be high.

The algorithms comparison exposed is also based on their execution times. The supervised methods with texture patterns have a training time necessary to achieve the best parameters for class discrimination. It is worth to mention that SVM classifier involves an optimization process to improve decision boundary, for this reason time measurement in this case becomes greater. Features extraction time for supervised 
methods are considerably higher in contrast with algorithm based on area, almost 14 times slower, due to images division, gray level co-occurrence matrices and statistical measures of texture calculations, however, with features obtained, KNN and SVM classifiers are up to 14 times faster to discriminate the information on the feature space.

It can be thus being suggested that low level features extraction with thresholding classifier is the most appropriate method to be implemented in a removal robotic application, due to accuracy response and execution time. The other approaches despite having indices slightly better, result very slow for scene process.

One of the issues that emerge from the results is the possibility of the methods purposed to evaluate image crops in different stages of growth, this also accords with descriptors used for classes detection, hence, thresholding classifier requires a periodical weed removal tasks, therefore, weed size is smaller than the vegetables crops, thus, area feature is preserved and the evaluation is carried out in appropriate conditions. On the other hand, for texture-based classification, the weed area is not as relevant as low-level features, due to window evaluation and the descriptors related to local properties as spatial arrangement, color or intensity distribution. Another limitation lies in sunlight; this is a strong factor for classifiers performance. In spite of the fact that the present work uses outdoor images, the dataset was acquired avoiding overexposure, otherwise, green color of plants would tend to be white and the brightness increased, then, the approaches reviewed in this paper decrease the classification performance, because of color and intensity values for segmentation and texture measurements would not be homogeneous for each object in the scene. It is important to highlight that further experimentation will include a light control system on image acquisition stage.

The algorithms could be adopted to another crop type, in Thresholding detection, the descriptors refer to color and area, therefore, a different crop that keeps approach of weed size smaller than vegetables, through periodical maintenance, results in a suitable candidate for weed classification. In contrast, to use K$\mathrm{NN}$ and SVM classifiers based on texture features, the descriptors must be evaluated for the new crop, this means, review the class differences in feature space and the number of principal components to use for training.

For selective treatment of weed, the system should be in real time. To achieve this goal and suggesting as further work, decrease image size and use other shape features for thresholding classifier with the purpose of increase its indices performance. Meanwhile, use less texture features where most variance be retained between classes, trying other combinations of statistical texture measures, could improve classifiers performance, fully exploiting the evaluation time of unsupervised classifiers, which exposed the best accuracy percentages.

\section{Acknowledgements}

This work is supported by the project ING-1758 namely "Sistema de detección de malezas, suelo compacto, suelo seco, plagas y piedras en los campos de cultivo Fase 1", funded by the Research Vice rectory of the Nueva Granada Military University in Bogotá, Colombia.

\section{References}

[1] A. K. Shinde and M. Y. Shukla, "Crop detection by machine vision for weed management," International Journal of Advances in Engineering \& Technology, vol. 7, no. 3, p. 818, 2014.

[2] H. Y. Jeon, L. F. Tian, and H. Zhu, "Robust crop and weed segmentation under uncontrolled outdoor illumination,” Sensors, vol. 11, no. 6, pp. 6270-6283, 2011.

[3] D. Woebbecke, G. Meyer, K. Bargen, and D. Mortensen, "Color indices for weed identification under various soil, residue and lighting conditions," Transactions of the ASAE, vol. 38, no. 1, pp. 259-269, 1995.

[4] A. Perez, F. Lopez, J. Benlloch, and S. Christensen, "Colour and shape analysis techniques for weed detection in cereal fields," Computer and Electronics in Agriculture, vol. 25, no. 3, pp. 197-212, 2000.

[5] M. Mustafa, A. Hussain, K. Ghazali, and S. Riyadi, "Implementation of image processing technique in real time vision system for automatic weeding strategy," in Proc. IEEE International Symposium on Signal Processing and Information Technology, 2007, pp. 632-635. 
[6] L. Tang, B. Tian, B. Steward, and J. Reid, "Texture-based weed classification using Gabor wavelets and neural network for real-time selective herbicide applications," Urbana, vol. 51, p. 61801, 1999.

[7] A. Ishak, A. Hussain, and M. Mustafa, "Weed image classification using Gabor wavelet and gradient field distribution," Computers and Electronics in Agriculture, vol. 66, no. 1, pp. 53-61, 2008.

[8] T. Burks, S. Shearer, and F. Payne, "Classification of weed species using color texture features and discrimant anlysis," ASAE, vol. 43, pp. 441-448, 2000.

[9] V. Kovalev and S. Volmer, "Color co-ocurrence descriptors for querying-by-example," in Proc. Multimedia Modeling, 1998. MMM'98, 1998, pp. 32-38.

[10] S. Shearer and R. Holmes, "Plant identification using color co-ocurrence matrices," ASAE, vol. 33, no. 6, pp. 2037-2044, 1990.

[11] T. Burks, "Color image texture analysis and neural network classification of weed species," Ph.D. thesis, Biological and Agricultural Engineering, University of Kentucky, 1997.

[12] G. McLachlan, Discriminant Analysis and Statistical Pattern Recognition. New Jersey: John Wiley \& Sons, 2004.

[13] W. Wong, C. Ali, K. Brandon, C. Wee, and M. Marriapan, "Co-occurrence matrix with neural network classifier for weed species classification: A comparison between direct application of co-occurrence matrix (GLCM) and Haralick features as inputs," International Journal of Enhanced Research in Science Technology \& Engineering, vol. 2, no. 2, pp. 1-6, 2013.

[14] R. Haralick, K. Shanmugam, and I. Dinstein, "Textural features for image classification," IEEE Transactions on Systems, Man and Cybernetics, vol. 3, no. 6, pp. 610-621, 1973.

[15] G. Meyer and J. Neto, "Verification of color vegetation indices for automated crop imaging applications," Computers and Electronics in Agriculture, vol. 63, pp. 282-293, 2008.

[16] J. Du, X. Wang, and G. Zhang, "Leaf shape based plant species recognition," Applied Mathematics and Computation, vol. 185, no. 2, pp. 883-893, 2007.

[17] L. Wu and Y. Wen, "Weed/corn seedling recognition by support vector machine using texture features," African Journal of Agricultural Research, vol. 4, no. 9, pp. 840-846, 2009.

[18] P. Soille, Morphological Image Analysis: Principles and Applications. Springer Science \& Business Media, 2013.

[19] J. L. Semmlow and B. Griffel, Biosignal and Medial Image Processing. CRC Press, 2014.

[20] G. Shapiro, Computer and Robot Vision. Addison-Wesley, 1992.

[21] R. Kumar, K. Ramareddy, and B. Rao, "A simple region descriptor based on object area per scan line," International Journal of Computer Applications, vol. 3, no. 7, pp. 24-27, 2010.

[22] C. Pulido, M. Molina, and L. Solaque, "Machine vision system for weed detection using image filtering in vegetables crops," Revista Facultad de Ingeniería, vol. 80, pp. 4-8, 2016.

[23] B. Nie, J. Du, H. Liu, G. Xu, Z. Wang, Y. He, and B. Li, "Crowds' classification using hierarchical cluster, rough sets, principal component analysis and its combination," in International Forum on Computer Science-Technology and Applications, 2009, IFCSTA'09, vol. 1, pp. 287-290.

[24] T. Reddy, K. Devi, and S. Gangashetty, "Nonlinear principal component analysis for seismic data compression," in 1st Int'l Conf. on Recent Advances in Information Technology, RAIT-2012, pp. 927-932.

[25] Z. Lihong and G. Zikui, "Face recognition method based on adaptively weighted block-two dimensional principal component analysis," in Third International Conference on Computational Intelligence, Communication Systems and Networks, 2011, pp. 22-25.

[26] M. Richardson, Principal Component Analysis, Mathematical Modelling and Scientific Computing. University of Oxford, 2009.

[27] W. Henley and D. Hand, "A k-nearest-neighbour classifier for assessing consumer credit risk," The Statistician, pp. 77-95, 1996.

[28] T. Cover and P. Hart, "Nearest neighbor pattern classification," IEEE Transactions on Information Theory, vol. 13, no. 1, pp. 21-27, 1967.

[29] V. Garcia, E. Debreuve, and M. Barlaud, "Fast k nearest neighbor search using GPU," in Computer Vision and Pattern Recognition Workshops, 2008.

[30] X. Wu, V. Kumar, J. Quinlan, J. Ghosh, Q. Yang, H. Motoda, G. McLachlan, A. Ng, B. Liu, P. Yu, Z. Zhou, M. Steinbach, D. Hand, and D. Steinberg, "Top 10 algorithms in data mining," Knowl Inf Syst, vol. 14, no. 1, pp. 1-37, 2008.

[31] E. Osuna, F. Robert, and G. Federico. (1997). Support Vector Machines: Training and Applications. MIT and Center for Biological and Computational Learning. 
[32] J. Lagarias, J. Reeds, M. Wright, and P. Wright, "Convergence properties of the Nelder-Mead simplex method in low dimensions," SIAM Journal of Optimization, vol. 9, no. 1, pp. 112-147, 1998. 